\title{
Pneumococcal vaccination: time to move on?
}

\author{
Marijke Johanna Proesmans
}

Received: 28 April 2010 /Accepted: 29 April 2010 /Published online: 15 May 2010

(C) Springer-Verlag 2010

The search for an efficient pneumococcal vaccine has been driven by the enormous clinical impact of pneumococcal infections especially in young children. Streptococcus pneumoniae is the primary bacterial cause of childhood acute otitis media, bacteremia, pneumonia, and meningitis and is an important cause of mortality in the developing world [7]. The threat posed by $S$. pneumoniae is particularly high in certain risk groups: children below the age of 2 years, immune-compromised patients including HIV infection and patients with hematologic malignancies, diabetes, and severe renal failure. The spread of antimicrobial-resistant $S$. pneumoniae worldwide has added to the need for vaccine development $[7,8,12]$.

Pneumococcal polysaccharide antigens are unable to generate an efficient immune response in children younger than 2 years of age; pneumococcal polysaccharide vaccines are thus not protective in this age group [17, 22]. Conjugation or 'coupling' the pneumococcal polysaccharide antigen to an immunogenic protein carrier converts the polysaccharide antigen to a $\mathrm{T}$ cell-dependent antigen and alters the response to the polysaccharide in several important ways [20]: an efficient antibody response can be generated from a young age including development of memory B cells primed and ready to respond to either the polysaccharide (as would be encountered during an infection) or to a second dose of the same vaccine antigen. The development of pneumococcal conjugate vaccines allows the prevention of pneumococcal

M. J. Proesmans $(\bowtie)$

Department of Pediatrics, Cystic Fibrosis Reference Center,

University Hospital Leuven,

Herestraat 49,

3000 Leuven, Belgium

e-mail: Marijke.Proesmans@uzleuven.be disease in populations that are unable to generate an adequate immune response to polysaccharide vaccines.

Initial reports of decrease in invasive pneumococcal disease (IPD) after the introduction of the 7-valent pneumococcal conjugate vaccine (PCV-7) were impressive $[1,2,11$, 23]. In 2001, 1 year after the vaccine's license, the rate of IPD in children under 2 years of age dropped from 188 to 59 per 100,000 in the US $(-69 \% ; p<0.001)$; the rate of disease caused by vaccine and vaccine-related serotypes declined by $78 \%(p<0.001)$ and 50\% $(p<0.001)$, respectively [23]. Disease rates also fell in other age group including adults, a phenomenon called herd immunity.

Because of the limited number of serotypes contained in the PCV-7 vaccine, possible serotype replacement was anticipated and active surveillance was put in place in several countries including the US. The 'Center for Disease Control' and several groups have documented that the number of pneumococcal infections caused by non PCV7 vaccine serotypes is increasing, primarily serotype 19A [3, 9, 13, 15, 16, 18, 19]. Moreover, multidrug antibiotic resistance among the 19A serotype isolates has become a major concern [13-16].

The study published in this issue of the European Journal of Pediatrics reports on the surveillance of pediatric invasive pneumococcal infections in a tertiary care pediatric center in Quebec (Canada) after government sponsored introduction of PCV-7 vaccine for children up to age 5 years in a $2+1$ schedule for infants [5]. Other reports mainly concern $3+1$ schedules. This rather small study confirms data from several other epidemiological studies on evolution of pneumococcal epidemiology underlining the value of well-performed monocentric surveillance. A weakness of this study is the absence of individual patient vaccination status as well as data on the general vaccination uptake in the province. Data are given as absolute IPD numbers and not compared with overall admission data. 
The overall reduction in IPD of $61.4 \%$ in the whole pediatric age group is similar to other reports. In the postvaccination period, children with IPD were older (mean age of 26 months versus 18.8 months), but the percentage of children with underlying conditions was not higher. Interesting in this study is the detailed documentation of the different types of IPD. Post-vaccination, the proportion of bacteremia decreased while pneumonia increased. Meningitis remained at around $12 \%$ of all IPD, the absolute number of cases thus slightly decreasing. Although from 2008, the absolute number of IPD is again on the rise, it is still well below the pre-vaccination level. The recent increase is however mainly due to non-vaccine serotype infection, with serotype 19A as the main culprit; again, these data are in line with other reports.

In a recent publication, eight US children's hospitals report on a prospective surveillance of invasive pneumococcal infections from 1994 to 2008, vaccination being started in 2000 [10]. By 2004, there was an overall decline of $65 \%$ in IPD cases in all age groups. Compared to the period before start of vaccination $(n=400-500)$, IPD number has decreased yearly from $2001(n=200)$ through 2004 (150), with numbers again rising from 2005 to 2008 $(n=200)$. Similarly, bacteremia decreased and pneumonia increased. For the period of 2007-2008, 19A accounted for $46 \%$ of all serotypes. These results compare well to the current Canadian report, only the relative proportion of children with underlying disease was higher after vaccine introduction.

Although the PCV7 vaccine holds an inherent risk for serotype replacement, it would be an oversimplification to point to PCV-7 vaccine as the one and only cause of the worrying serotype replacement. Some serotypes including 1 and 19A were already on the rise before the vaccine was introduced as has been documented in Korea and Israel $[4,6]$. A Belgian survey also documented a rise in 19A before the introduction of vaccination with a further increase post-vaccination (unpublished data, manuscript in preparation). Interestingly, in the survey of Kaplan et al., there was an association between the number of PCV7 doses received before the infection and the likelihood of 19A being responsible [10]. In the current report, however, the proportion of $19 \mathrm{~A}$ was almost as high $(40.7 \%)$ in the context of a $2+1$ schedule compared to a $3+1$ schedule.

So where are we now? PCV7 is immunogenic and highly protective against vaccine serotypes in a $3+1$ as well as a $2+1$ schedule in young children. Large scale vaccination has decreased the total number of IPD substantially. Unfortunately, for meningitis, the IPD with the highest mortality and morbidity, the vaccine has not made a big difference. Although recently the IPD incidence is rising, it is still well below the pre-vaccine level. Data are however worrying in that very virulent serotypes like 19A are on the rise, even more so because of the high antibiotic resistance of this specific serotype. It is therefore time that the new pneumococcal vaccines become available, especially the 13 valent one since it includes the latter serotype. Further surveillance remains of utmost importance. In addition, further research towards a pneumococcal vaccine based on a common pneumococcal protein may be needed in order to obtain a consistent and lasting decrease in IPD [21].

\section{References}

1. Black S, France EK, Isaacman D et al (2007) Surveillance for invasive pneumococcal disease during 2000-2005 in a population of children who received 7-valent pneumococcal conjugate vaccine. Pediatr Infect Dis J 26(9):771-777

2. Black S, Shinefield H, Fireman B et al (2000) Efficacy, safety and immunogenicity of heptavalent pneumococcal conjugate vaccine in children. Northern California Kaiser Permanente Vaccine Study Center Group. Pediatr Infect Dis J 19(3):187-195

3. Byington CL, Korgenski K, Daly J et al (2006) Impact of the pneumococcal conjugate vaccine on pneumococcal parapneumonic empyema. Pediatr Infect Dis J 25(3):250-254

4. Choi EH, Kim SH, Eun BW et al (2008) Streptococcus pneumoniae serotype 19A in children, South Korea. Emerg Infect Dis 14 (2):275-281

5. Crisinel, PA, I Chevalier, F Rallu et al (2010) Invasive pneumococcal disease after implementation of a reduced three-dose pneumococcal conjugate vaccine program: a pediatric tertiary care center experience. Eur J Ped 169(11):1311-1315

6. Dagan R, Givon-Lavi N, Leibovitz E et al (2009) Introduction and proliferation of multidrug-resistant Streptococcus pneumoniae serotype 19A clones that cause acute otitis media in an unvaccinated population. J Infect Dis 199(6):776-785

7. Eskola J, Takala AK, Kilpi TM et al (1998) Clinical evaluation of new pneumococcal vaccines: the Finnish approach. Dev Biol Stand 95:85-92

8. Greenwood B (1999) The epidemiology of pneumococcal infection in children in the developing world. Philos Trans R Soc Lond B Biol Sci 354(1384):777-785

9. Hicks LA, Harrison LH, Flannery B et al (2007) Incidence of pneumococcal disease due to non-pneumococcal conjugate vaccine (PCV7) serotypes in the United States during the era of widespread PCV7 vaccination, 1998-2004. J Infect Dis 196 (9):1346-1354

10. Kaplan SL, Barson WJ, Lin PL et al (2010) Serotype 19A is the most common serotype causing invasive pneumococcal infections in children. Pediatrics 125(3):429-436

11. Kaplan SL, Mason EO Jr, Wald ER et al (2004) Decrease of invasive pneumococcal infections in children among 8 children's hospitals in the United States after the introduction of the 7-valent pneumococcal conjugate vaccine. Pediatrics 113(3 Pt 1):443-449

12. McIntyre P (1997) Epidemiology and prevention of pneumococcal infections. Curr Opin Pediatr 9(1):9-13

13. Messina AF, Katz-Gaynor K, Barton T et al (2007) Impact of the pneumococcal conjugate vaccine on serotype distribution and antimicrobial resistance of invasive Streptococcus pneumoniae isolates in Dallas, TX, children from 1999 through 2005. Pediatr Infect Dis J 26(6):461-467

14. Moore MR, Gertz RE Jr, Woodbury RL et al (2008) Population snapshot of emergent Streptococcus pneumoniae serotype 19A in the United States, 2005. J Infect Dis 197(7):1016-1027 
15. Pai R, Moore MR, Pilishvili T et al (2005) Postvaccine genetic structure of Streptococcus pneumoniae serotype 19A from children in the United States. J Infect Dis 192(11):1988-1995

16. Pelton SI, Huot H, Finkelstein JA et al (2007) Emergence of $19 \mathrm{~A}$ as virulent and multidrug resistant Pneumococcus in Massachusetts following universal immunization of infants with pneumococcal conjugate vaccine. Pediatr Infect Dis J 26 (6):468-472

17. Poland GA (1999) The burden of pneumococcal disease: the role of conjugate vaccines. Vaccine 17(13-14):1674-1679

18. Singleton RJ, Hennessy TW, Bulkow LR et al (2007) Invasive pneumococcal disease caused by nonvaccine serotypes among alaska native children with high levels of 7 -valent pneumococcal conjugate vaccine coverage. JAMA 297(16):1784-1792
19. Steenhoff AP, Shah SS, Ratner AJ et al (2006) Emergence of vaccine-related pneumococcal serotypes as a cause of bacteremia. Clin Infect Dis 42(7):907-914

20. Stein KE (1992) Thymus-independent and thymus-dependent responses to polysaccharide antigens. J Infect Dis 165(Suppl 1): S49-S52

21. Tai SS (2006) Streptococcus pneumoniae protein vaccine candidates: properties, activities and animal studies. Crit Rev Microbiol 32(3):139-153

22. Torzillo PJ (1997) Pneumococcal vaccine. Aust Fam Physician 26 (8):937-941

23. Whitney CG, Farley MM, Hadler J et al (2003) Decline in invasive pneumococcal disease after the introduction of proteinpolysaccharide conjugate vaccine. N Engl J Med 348(18):17371746 